\title{
Bragg magnifier: A detector for submicrometer x-ray computer tomography
}

Marco Stampanonia)

Institute of Biomedical Engineering, ETH and University of Zürich, Moussonstrasse 18, CH-8044 Zurich, Switzerland and Swiss Light Source, Paul Scherrer Institute, CH-5232 Villigen, Switzerland

Gunther Borchert

Institut für Kernphysik, Forschungszentrum Jülich, D-52425 Jülich, Germany

Rafael Abela

Swiss Light Source, Paul Scherrer Institute, CH-5232 Villigen, Switzerland

Peter Rüegsegger

Institute of Biomedical Engineering, ETH and University of Zürich, Moussonstrasse 18, CH-8044 Zürich, Switzerland

(Received 20 June 2002; accepted 19 September 2002)

$\mathrm{X}$-ray computer microtomography is a powerful tool for nondestructive examinations in medicine, biology, and material sciences. The resolution of the presently used detector systems is restricted by scintillator properties, optical light transfer, and charge-coupled-device granularity, which impose a practical limit of about $1 \mu \mathrm{m}$ spatial resolution at detector efficiencies of a few percent. A recently developed detector, called the Bragg magnifier, achieves a breakthrough in this respect, satisfying the research requirements that ask for an efficient advance towards the submicron range. The Bragg magnifier uses the properties of asymmetric Bragg reflections to increase the cross section of the diffracted x-ray beam. Magnifications of up to $100 \times 100$ can be achieved even at hard $\mathrm{x}$-rays energies $(>20 \mathrm{keV})$. In this way, the influence of the detector resolution can be scaled down accordingly and the efficiency increased. Such a device has been developed and successfully integrated into the Microtomography Station of the Materials Science Beamline of the Swiss Light Source. The device is operated at energies ranging from 21.1 to $22.75 \mathrm{keV}$, reaching spatial resolutions of $140 \mathrm{~nm}$, and is ready for submicrometer microtomography. (C) 2002 American Institute of Physics. [DOI: 10.1063/1.1520722]

\section{INTRODUCTION}

The necessity to achieve micrometer or even submicrometer spatial resolution in hard $\mathrm{x}$-ray $(>20 \mathrm{keV})$ synchrotron-based computed tomography $(\mathrm{SR} \mu \mathrm{CT})$ has spurred the development of detectors optimized for spatial resolutions. The most established detection method in SR $\mu \mathrm{CT}$ consists of converting $\mathrm{x}$ rays into visible light with a scintillator and projecting them onto a charge-coupled-device (CCD) with the help of suitable microscopic optics. ${ }^{1-4}$ Stateof-the-art detectors offer $0.8 \mu \mathrm{m}$ resolution. Efficiency, however, with $0.5 \%$ at $30 \mathrm{keV}$, is very low. ${ }^{5}$ Alternative approaches have been suggested to trespass this threshold: Fresnel zone plates ${ }^{6}$ and parabolic compound refractive lenses ${ }^{7}$ have been demonstrated to reach submicrometer resolution $(0.3 \mu \mathrm{m})$, but they can be efficiently used only at energies well below $20 \mathrm{keV}$.

In this work, a different approach is presented to efficiently achieve the submicrometer range for hard $\mathrm{x}$ rays. The instrument, called Bragg magnifier, is based on the wellknown principle of asymmetrical Bragg diffraction, but it has been designed to work at high energies $(>20 \mathrm{keV})$ and to be easily integrated in a microtomography device. Bragg diffraction from an asymmetrically cut crystal produces onedimensional magnification. A second diffraction from an-

${ }^{a)}$ Author to whom correspondence should be addressed; electronic mail: stampanoni@biomed.ee.ethz.ch other crystal with the same magnification factor but perpendicular diffraction plane produces uniform twodimensional (2D) magnification (see Figs. 1 and 2). Kuriyama et al. ${ }^{8}$ operated such a setup at $12.3 \mathrm{keV}$ and resolved approximately $420 \mathrm{lp} / \mathrm{mm}$. Our instrument is designed to reach $2500 \mathrm{lp} / \mathrm{mm}$ at $22.75 \mathrm{keV}$. It has been shown ${ }^{9}$ that this kind of x-ray optics can produce absorption, edgeenhanced, or phase-contrast images.

\section{THEORY AND SIMULATION}

The x-ray optics of the Bragg magnifier consist of two asymmetrically cut silicon crystals in Kirkpatrik-Baez geometry, see Fig. 1. Performances and constrains of the Bragg magnifier have been estimated with the help of dynamical $\mathrm{x}$-ray diffraction theory ${ }^{10-12}$ and ray-tracing simulations. ${ }^{13}$ One of the most important parameters to be determined is the width of the Darwin curve for asymmetric diffraction (incident and reflected) since the precision of the mechanics that manipulates the crystals has to be designed accordingly. In addition, the angle of incidence and the angle of reflection, as well as the magnification factor, have to be known. In the following, we only consider $\sigma$-polarized $\mathrm{x}$ rays as emerging from a synchrotron wiggler. The width of the diffraction pattern for a nonabsorbing perfect crystal is given by ${ }^{14}$

$$
\omega_{s}=\frac{2}{\sin \left(2 \theta_{B}\right)} \frac{r_{0} \lambda^{2}}{\pi V}\left|F_{h}\right| e^{-\mathrm{DW}},
$$




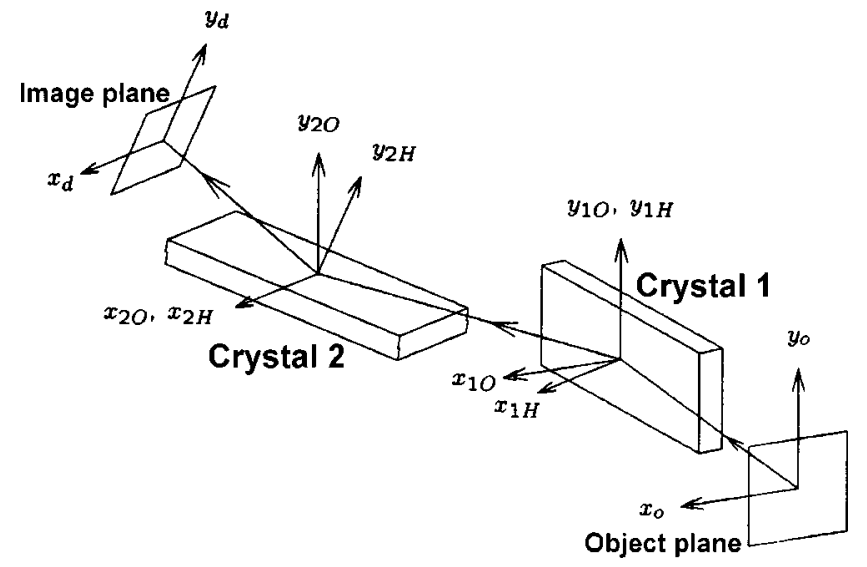

FIG. 1. X-ray optics of the Bragg magnifier showing the main crystals and the object and image (detector) planes. " $O$ " describes the incident beam coordinates, " $H$ " the diffracted one. Adapted from Ref. 9.

where $r_{0}=2.818 \times 10^{-15} \mathrm{~m}$ is the classical electron radius, $\lambda$ is the wavelength of the incident radiation, $V$ is the volume of the unit cell, $\theta_{B}$ is the Bragg angle, $F_{h}$ is the crystal structure factor, and $e^{-\mathrm{DW}}$ is the Debye-Waller factor. For real crystals, whose diffraction planes are cut at an asymmetry angle $\alpha$ with respect to the surface, absorption and asymmetry factors cannot be neglected. Absorption is described by the imaginary part of the complex susceptibility $\chi$ and the asymmetry factor is related to the magnification factor $m$ by

$$
m=\frac{\sin \left(\theta_{B}+\alpha\right)}{\sin \left(\theta_{B}-\alpha\right)} .
$$

Following Ref. 15, the Darwin curve is given by

$$
R_{H}(y)=L-\sqrt{L^{2}-1},
$$

where

$$
\begin{aligned}
L= & \frac{\chi_{H r}^{2}}{\chi_{H r}^{2}+\chi_{H i}^{2}}\left[y^{2}+g^{2}\right. \\
& \left.+\sqrt{\left(y^{2}-g^{2}-1+\frac{\chi_{H i}^{2}}{\chi_{H r}^{2}}\right)^{2}+4\left(g y-\frac{\chi_{H i}}{\chi_{H r}}\right)^{2}}\right],
\end{aligned}
$$

with

$$
y=\frac{(m-1) \sqrt{|m|}}{2 C m} \frac{\chi_{0 r}}{\left|\chi_{H r}\right|}+\frac{\sqrt{|m|}\left(\theta_{0}-\theta_{B}\right) \sin \left(2 \theta_{B}\right)}{m C\left|\chi_{H r}\right|},
$$

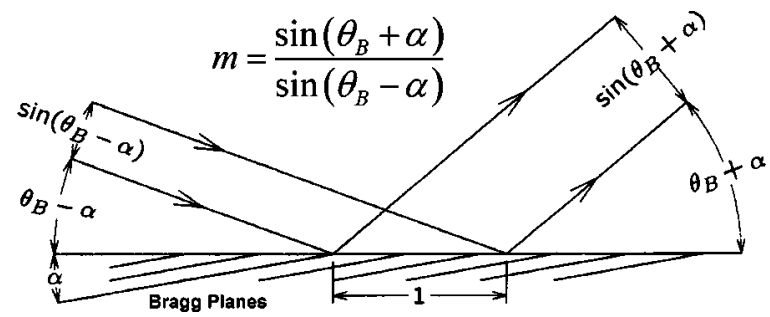

FIG. 2. Coplanar asymmetric one-dimensional Bragg diffraction. The magnification direction lies in the plane of diffraction defined by the incident wave vector and the Bragg vector. $m$ is the magnification factor. Adapted from Ref. 9.

$$
g=\frac{(m-1) \sqrt{|m|}}{2 C m} \frac{\chi_{0 i}}{\left|\chi_{H r}\right|} .
$$

From Eq. (5), the angle $\theta_{0}$ between the incidence direction and the diffraction planes can be obtained directly and the angle $\theta_{H}$ can be obtained just by replacing $m$ by $1 / m$ :

$$
\begin{aligned}
& \theta_{0}=\theta_{B}+(1+m) \frac{\left|\chi_{0 r}\right|}{2 \sin \left(2 \theta_{B}\right)}+\sqrt{|m|} \frac{\left|\chi_{H r}\right|}{\sin \left(2 \theta_{B}\right)} y, \\
& \theta_{H}=\theta_{B}+\left(1+\frac{1}{m}\right) \frac{\left|\chi_{0 r}\right|}{2 \sin \left(2 \theta_{B}\right)}+\frac{1}{\sqrt{|m|}} \frac{\left|\chi_{H r}\right|}{\sin \left(2 \theta_{B}\right)} y .
\end{aligned}
$$

In the asymmetric case $(|m| \neq 1)$ the intensity distribution $R_{H}\left(\theta_{0}\right)$ is different from $R_{H}\left(\theta_{H}\right)$. The diffracted intensity has an appreciable value only in the range $-1 \leqslant y \leqslant 1$, which corresponds to an angular acceptance of

$$
\omega_{0}=\sqrt{|m|} \omega_{s},
$$

and to an angular range of emergence of

$$
\omega_{H}=\frac{1}{\sqrt{|m|}} \omega_{s} .
$$

In addition, the center of the diffraction curve is shifted from the Bragg angle by

$$
\begin{aligned}
& \delta \theta_{0}=\frac{1}{2}(1+m) \delta \theta_{s}, \\
& \delta \theta_{H}=\frac{1}{2}[1+(1 / m)] \delta \theta_{s},
\end{aligned}
$$

where $\delta \theta_{s}$ is the deviation from the Bragg law in the symmetric case given by

$$
\delta \theta_{s}=\frac{\chi_{0 r}}{\sin \left(2 \theta_{B}\right)}=\frac{1}{\sin \left(2 \theta_{B}\right)} \frac{r_{0} \lambda^{2}}{\pi V} F_{0 r} .
$$

Both angular divergences of the incident and reflected beam as well as their cross section are related to each other by the magnification $m$. In fact, it holds that

$$
\omega_{H}=\frac{1}{m} \omega_{0},
$$

and for $S_{0}$ and $S_{H}$, being the spatial cross sections of the incident and diffracted beams, Liouville's theorem requires that $S_{0} \omega_{0}=S_{H} \omega_{H}$, and hence,

$$
S_{H}=|m| S_{0} .
$$

As a consequence, the "Fankuchen" effect can be observed, i.e., if $|m|>1$ the range of total reflection for the emergent beam is $1 /|m|$ times smaller than that of the incident beam, while its spatial cross section is $|m|$ times greater. According to these theoretical considerations, several combinations of asymmetry angles and lattice planes have been investigated in order to match the requirements of the experiment. $\operatorname{Si}(220)$ with an asymmetry angle of $\alpha=8.0^{\circ}$ resulted in being the best candidate for the magnifying optics. Figure 3 represents the Darwin curves of $\mathrm{Si}(220)$ for an energy of $21.75 \mathrm{keV}$ and $\alpha=8.0^{\circ}$. The magnification factor is $m$ $=30.4$ and the full width at half maxima (FWHMs) of the rocking curve are $\omega_{0}=10.15^{\prime \prime}$ and $\omega_{H}=0.34^{\prime \prime}$ for the inci- 


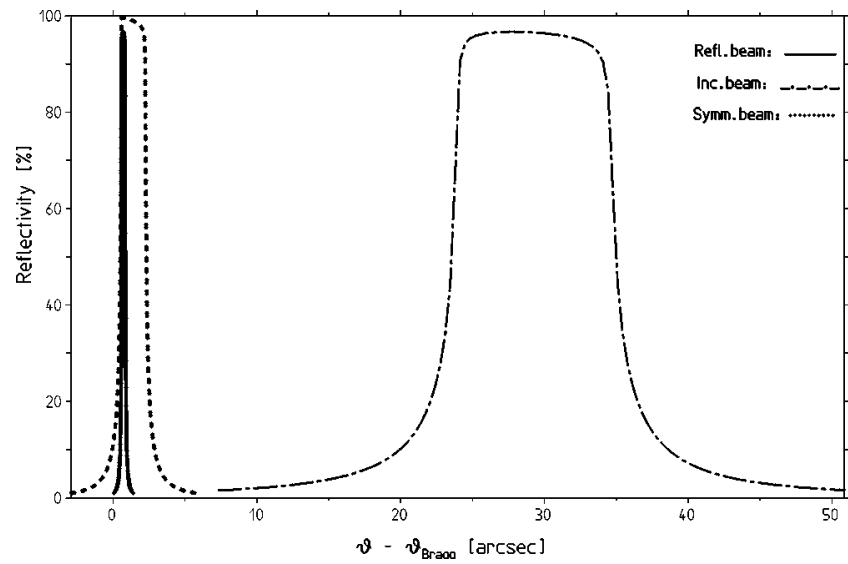

FIG. 3. Diffraction properties of $\mathrm{Si}(220)$ for an x-ray energy of $21.75 \mathrm{keV}$ (Bragg angle $\theta_{B}=8.536^{\circ}$ ) and an asymmetry angle of $8.0^{\circ}$. Rocking curves for symmetric as well as for the asymmetric case are plotted. Their FWHMs are $1.84^{\prime \prime}, 10.15^{\prime \prime}$, and $0.34^{\prime \prime}$ respectively.

dent and reflected beams, respectively. Table I summarizes these parameters for different energies. The main optical components of the Materials Sciences Beamline 4S (see Fig. 4 and Ref. 16) have been extensively simulated with the ray-tracing program SHADOW ${ }^{13}$ in order to find out the optimal beamline settings for tailoring a x-ray beam suited for the Bragg magnifier. In a previous work, ${ }^{17}$ it has been shown that the beamline optics can deliver a beam with $21-23 \mathrm{keV}$ with less than $20 \mu \mathrm{rad}$ of divergence. The corresponding beamline settings are further on called the "collimated beam delivery modus." A comparison with the relevant rocking curve implies that the first $\mathrm{Si}(220)$ Bragg crystal will accept $95 \%$ of the incoming intensity. The results of those simulations suggested that with such a setup a linear amplification of a factor of at least $30 \times$ in both dimensions is feasible. The resulting $\mathrm{x}$-ray magnified image can be converted to visible light and conveyed to the CCD in a much more efficient way (by the use of a very thick scintillator and a suitable optics) without deterring the final spatial resolution.

\section{REALIZATION}

The silicon crystals have been cut and prealigned with an asymmetry angle of $8.0^{\circ}$ with respect to the (220) planes

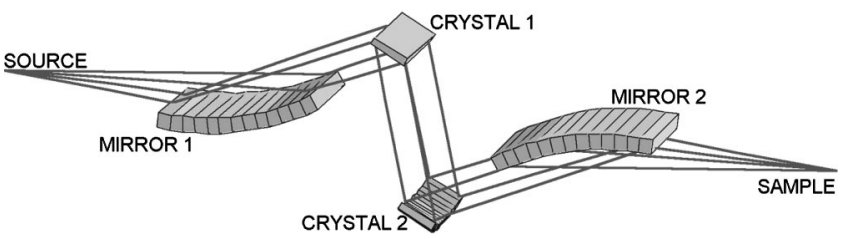

FIG. 4. Optics of the Materials Science Beamline MS: vertical collimating mirror (mirror 1), first monochromator flat crystal (crystal 1), second monochromator crystal for horizontal focusing (crystal 2) and vertical focusing mirror (mirror 2). For collimated beam delivery crystal 2 and mirror 2 are not bent.

(Holm, Thann, Germany). At the Institut für Kristallzüchtung in Berlin, the preorientation has been checked by means of a Laue diagram, using as a reference the 551 reflex. In fact, alignment with respect to the (220) reflex is difficult because of the large asymmetry angle. Therefore, the crystal was oriented with respect to the (551) reflex, which shows an offset of $0.5^{\circ}$ with respect to the (220) reflex, and the investigating $\mathrm{x}$-ray beam was oriented along the (110) direction. The crystals were fine cut with a $\mathrm{SiC}$ saw, with an angular accuracy of one arcminute and a surface roughness of better than 1 $\mu \mathrm{m}$. The asymmetry angle and the parallelism of the projection of the lattice normal with respect to the crystal surface could be obtained both with this accuracy. The backside of the crystals has been polished with a chemical-mechanical procedure (Cyton method), which eliminated all the residual saw dust. The crystals have been fixed by optical contacting on a glass support (Carl Zeiss, Germany). The glass support acts as a mechanical interface between the silicon crystal and the steel support (see below). In this way no mechanical stress is applied to the crystal when it is fixed on the goniometers, reducing dramatically any surface warping or distortions. The crystal-glass unit is mounted on a steel flange that is fixed on a double swivel. This system allows the crystal to be pitched and rolled with an angular reproducibility of 2 ". The crossed swivels are mounted on a high-resolution goniometer (Kohzu Precision, Co., Ltd., Japan), which rocks the crystal with an angular resolution of $0.05^{\prime \prime}$ in order to cope with the very narrow rocking curve (see Fig. 3). The second crystal is mounted in the same manner but rotated by $90^{\circ}$ with respect to the first one, and, in addition, it can be translated along the $Y$ and $Z$ directions. All the mechanical

TABLE I. Main parameters for $\mathrm{Si}(220)$ at different energies. $\omega_{s}, \omega_{0}, \omega_{H}$ are the FWHM of the rocking curves for the symmetric case, and for the impinging and reflected beam of the asymmetrical case respectively, see Eqs. (7) and (8).

\begin{tabular}{cccccc}
\hline \hline Energy $[\mathrm{keV}]$ & Bragg Angle $\left[{ }^{\circ}\right]$ & Magnification & $\omega_{s}\left[{ }^{\prime}\right]$ & $\omega_{0}\left[^{\prime \prime}\right]$ & $\omega_{H}\left[{ }^{\prime \prime}\right]$ \\
\hline 21.10 & 8.801 & 20.67 & 1.901 & 8.63 & 0.419 \\
21.50 & 8.636 & 25.78 & 1.866 & 9.46 & 0.368 \\
21.75 & 8.536 & 30.40 & 1.844 & 10.15 & 0.335 \\
21.95 & 8.458 & 35.44 & 1.827 & 10.86 & 0.308 \\
22.10 & 8.400 & 40.42 & 1.815 & 11.52 & 0.286 \\
22.22 & 8.354 & 45.51 & 1.805 & 12.16 & 0.268 \\
22.35 & 8.306 & 52.64 & 1.795 & 13.00 & 0.248 \\
22.39 & 8.291 & 55.30 & 1.792 & 13.30 & 0.241 \\
22.46 & 8.265 & 60.64 & 1.786 & 13.89 & 0.230 \\
22.60 & 8.213 & 75.08 & 1.775 & 15.36 & 0.205 \\
22.75 & 8.159 & 100.57 & 1.763 & 17.66 & 0.176 \\
\hline \hline
\end{tabular}




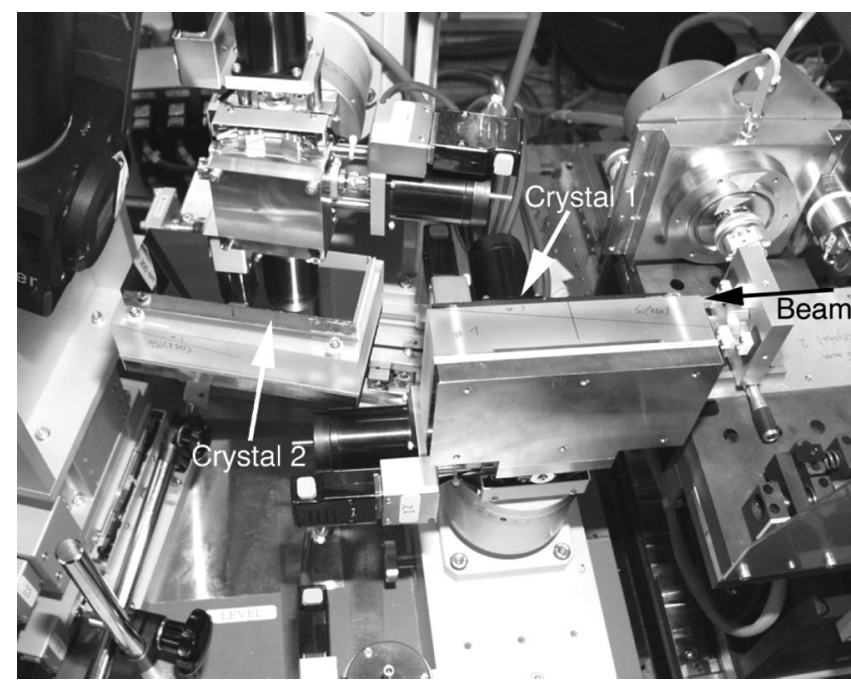

FIG. 5. Bragg magnifier setup at the Materials Science Beamline 4S. Visible are both crystals mounted on their swivels fixed to the high-resolution goniometers. The beam comes from the right. On the left side, the entrance of the $1: 1$ optic is also visible.

interfaces as well as swivels and goniometers have been manufactured in stainless steel, which guarantees high stability. Figure 5 depicts the Bragg magnifier installed at the Materials Science Beamline of the Swiss Light Source (SLS). Arrows show the two crystals as well as the direction of the impinging beam. The magnified $\mathrm{x}$-ray image is converted to visible light by a $35 \times 35 \mathrm{~mm}^{2} \mathrm{CsI}(\mathrm{Tl})$ scintillator of $300 \mu \mathrm{m}$ thickness. The generated light is collected by a highefficiency 1:1 tandem optic $(f=150 \mathrm{~mm}$, aperture $1 / 25)$ and projected onto the CCD. This optical system (Optique Peter, Lyon) can be translated along the $X$ and $Y$ directions and the scintillator support can accommodate scintillators of different sizes and thicknesses. The CCD camera (Pixel Vision of Oregon, Inc.), is equipped with a Thomson Th7899M fullframe chip with $2048 \times 2048$ pixels of $14 \mu \mathrm{m}$ pitch. A full frame can be read out in 100-250 ms through four independent outputs at 12-14 bit dynamical range and data are stored directly to the SLS file server via a fiber optic link. The camera has an optical shutter (open-close cycle of $30-40 \mathrm{~ms}$ ) to reduce the afterglow effect of the scintillator and to allow a completely dark readout.

\section{RESULTS}

The optics of the beamline $4 \mathrm{~S}$ were operated in the collimated beam delivery modus. This means that the second monochromator crystal as well as the second mirror are not bent, in order to deliver a low divergence beam $(<20 \mu \mathrm{rad})$. The energy was tuned from 21.1 up to 22.75 $\mathrm{keV}$, and the two crystals of the Bragg magnifier have been rocked accordingly. This produced magnification factors ranging from $20 \times 20$ to $100 \times 100$. Figure 6 shows the rocked angular position of the two crystals $\left(\varphi_{1}\right.$ for the first one, $\theta_{2}$ for the second) as a function of the energy. The $0^{\circ}$ direction (left axis in Fig. 6) corresponds to the prerocking position, i.e., when the surface of the first crystal is parallel to the impinging $x$-ray beam. The reproducibility of this energy scan was excellent: in fact, the error bars for the angular

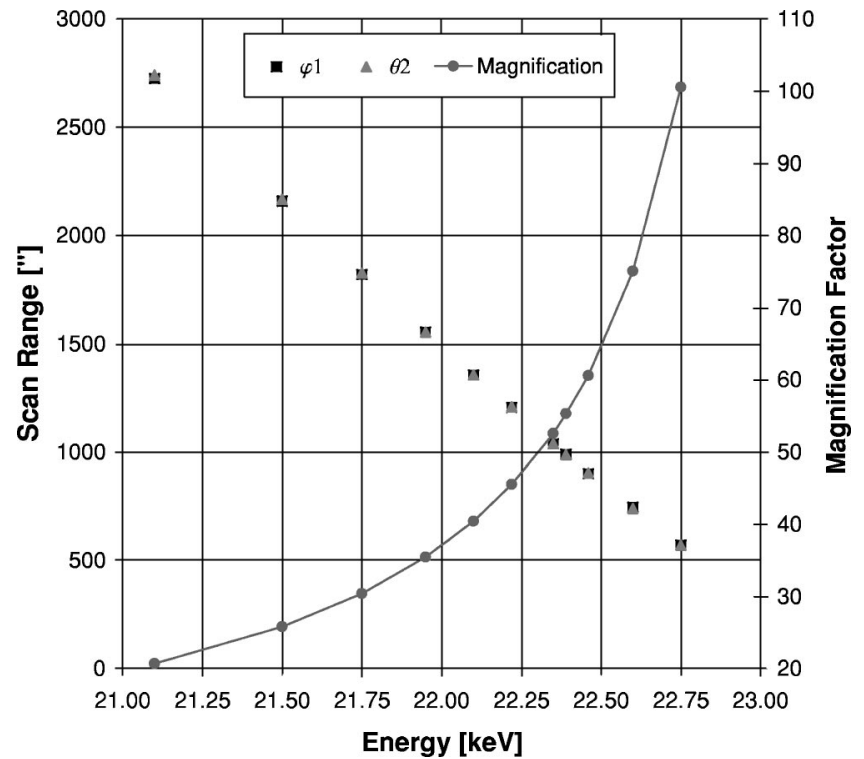

FIG. 6. Solid line shows the magnification factor as a function of the photon energy while the single markers give the positions of Bragg peaks $\varphi_{1}$ and $\theta_{2}$. The zero value on the left axis corresponds to the prerocking position, i.e., surface of the crystal and impinging beam are parallel. Error bars are smaller than the markers.

position are much smaller than the markers on the plot. These values, given in arcseconds, correspond to the angle $\beta$ of the incident beam with respect to the crystal surface defined by: $\beta=\theta_{B}-\alpha$, where $\alpha$ is the asymmetry angle. On the same plot (right axis) the theoretical magnification factor as a function of the energy is depicted. Figure 7 shows the excellent agreement between expected magnification, predicted by dynamical diffraction theory, and measured magnification, obtained with the help of a boron fiber (see Fig. 8) of known dimension $(100 \mu \mathrm{m}$ outer diameter and $15 \mu \mathrm{m}$ tungsten core). An image of the calibration fiber has been acquired at the same experimental conditions [ $\mathrm{x}$-ray energy of $22.1 \mathrm{keV}$, sample-to-scintillator distance (SSD) of 350 $\mathrm{mm}$, magnification $40 \times 40$ ] with the standard detector installed at the Materials Science Beamline, which has a spatial resolution of $250 \mathrm{lp} / \mathrm{mm}$ at $10 \%$ modulator transfer function (MTF) ${ }^{18}$ The direct comparison depicted in Fig. 8 shows the evident enhancement in terms of spatial resolution provided by the Bragg magnifier. By reducing the SSD to a few $\mathrm{mm}$, the x-ray optical resolution gain from Fig. 8(b) to 8(a) would look less splendid but, on the other hand, the Bragg magnifier allows much higher magnification factors as well as higher efficiency than the standard detector. An illustration of the imaging qualities of the Bragg magnifier is given in Fig. 9 , which shows a human bone trabecula together with the boron fiber with magnification factors of $20 \times 20,30 \times 30$, $40 \times 40,60 \times 60,75 \times 75$, and $100 \times 100$ : the corresponding field of view ranges from $1.44 \times 1.44$ to $0.28 \times 0.28 \mathrm{~mm}^{2}$ and the theoretical pixel size from $0.7 \times 0.7$ to $0.14 \times 0.14 \mu \mathrm{m}^{2}$.

\section{DISCUSSION AND OUTLOOK}

In this work the performance of an $\mathrm{x}$-ray microscope based on double asymmetric Bragg diffraction is described. The microscope works with hard $\mathrm{x}$ rays. If the normal to 


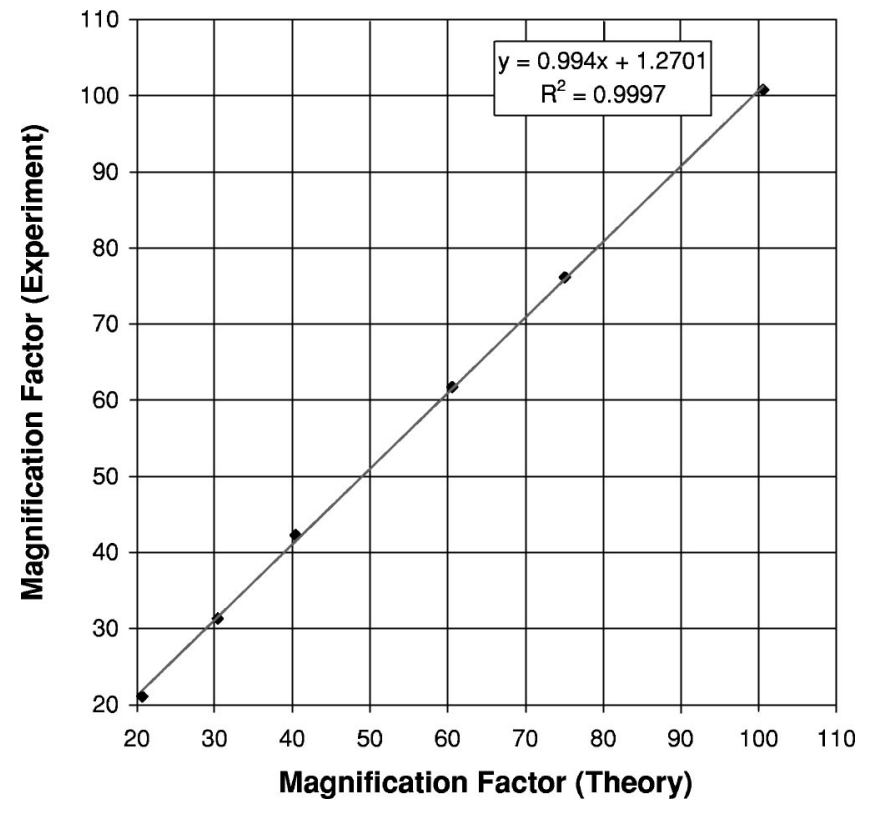

FIG. 7. Expected magnification, calculated with dynamical diffraction theory vs measured magnification, extracted with the help of the calibration fiber depicted in Fig. 9. The straight line with slope 1 indicates excellent agreement between theory and measurement.

diffraction plane $\mathbf{n}$ is correctly aligned with respect to impinging wave vector $\mathbf{k}$, i.e., if the incoming beam "sees" the correct asymmetry angle and not only its projection, the resulting image fidelity (see Fig. 9) is very good, and no distortions or optical aberrations could be detected. The Bragg magnifier has been operated with magnification factors ranging from $20 \times 20$ to $100 \times 100$, which corresponds to theoretical pixel sizes varying from $700 \times 700$ to $140 \times 140 \mathrm{~nm}^{2}$. With the 300- $\mu$ m-thick unstructured CsI(Tl) scintillator used so far, structures of less than $1 \mu \mathrm{m}$ can easily be detected. In the future, the unstructured $\mathrm{CsI}(\mathrm{Tl})$ scintillator shall be replaced by a structured one ${ }^{19}$ to provide an even higher spatial resolution at $\mathrm{x}$-ray detection efficiency higher than $90 \%$. The crystals have considerable dimensions $\left(150 \times 150 \mathrm{~mm}^{2}\right)$ because the microscope can accommodate fields of view (FOV) ranging from $1.44 \times 1.44$ to $0.28 \times 0.28 \mathrm{~mm}^{2}$ and because the Bragg angle differs from the asymmetry angle only by a small amount. This means that the distance between the
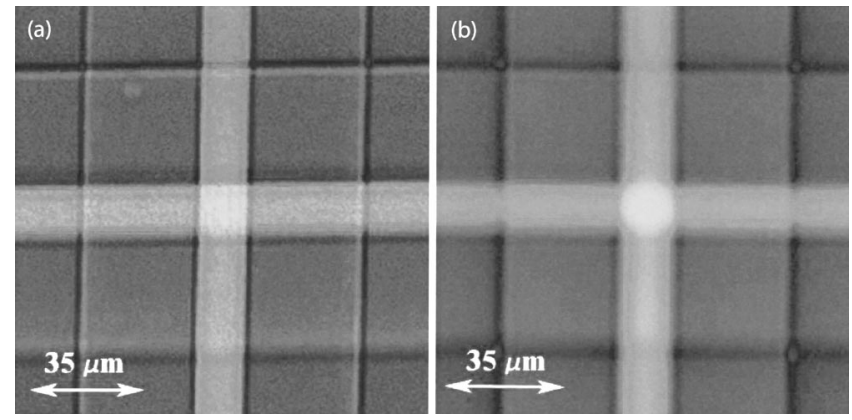

FIG. 8. Calibration fiber (100 $\mu \mathrm{m}$ boron outer diameter, $15 \mu \mathrm{m}$ tungsten core) acquired with the Bragg magnifier (a) and the standard detector (b) at the same $\mathrm{x}$-ray energy of $22.10 \mathrm{keV}$. The $\mathrm{x}$-ray propagation path sample scintillator was $350 \mathrm{~mm}$ in both cases. Resolution enhancement in (a) is clearly visible.
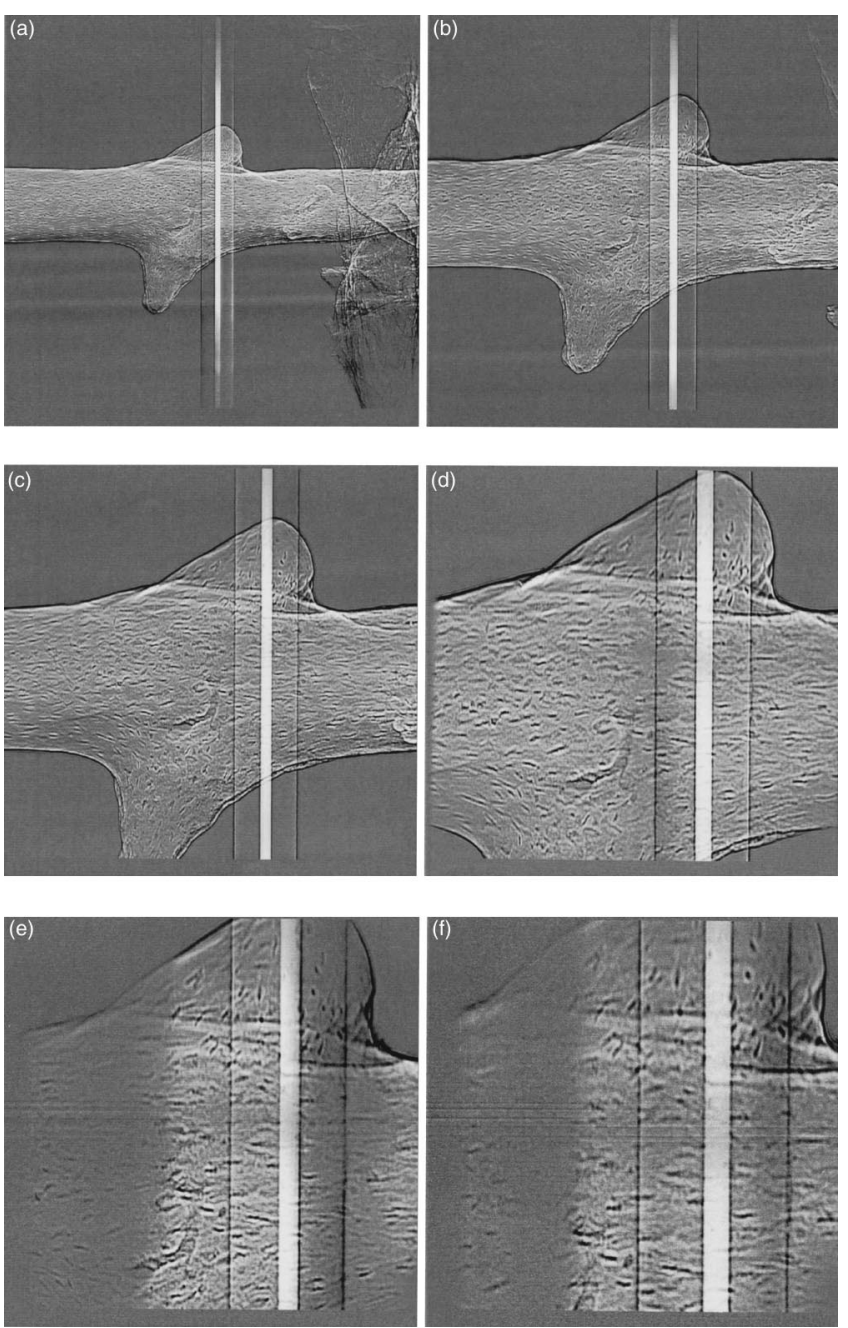

FIG. 9. Human bone trabecula together with a boron fiber of $100 \mu \mathrm{m}$ diameter and $15 \mu \mathrm{m}$ tungsten core imaged at different magnifications: (a) 20 $\times 20$, (b) $30 \times 30$, (c) $40 \times 40$, (d) $60 \times 60$, (e) $75 \times 75$, and (f) $100 \times 100$. On the right side of (a) the wax used for fixing the trabecula is visible. In (d), on the upper part of the trabecula border a diffraction fringe can be observed. An increased flux will allow a better alignment of the high-magnification configuration and (e) and (f) will appear complete and not cut on their left side. (a) Magnification: $20 \times 20$; FOV: $1.44 \times 1.44 \mathrm{~mm}^{2}$; and theoretical pixel size: $0.7 \times 0.7 \mu \mathrm{m}^{2}$. (b) Magnification: $30 \times 30$; FOV: 0.95 $\times 0.95 \mathrm{~mm}^{2}$; and theoretical pixel size: $0.46 \times 0.46 \mu \mathrm{m}^{2}$. (c) Magnification: $40 \times 40$; FOV: $0.72 \times 0.72 \mathrm{~mm}^{2}$; and theoretical pixel size: 0.35 $\times 0.35 \mu \mathrm{m}^{2}$. (d) Magnification: $60 \times 60$; FOV: $0.48 \times 0.48 \mathrm{~mm}^{2}$; and theoretical pixel size: $0.23 \times 0.23 \mu \mathrm{m}^{2}$. (e) Magnification: $75 \times 75$; FOV: $0.38 \times 0.38 \mathrm{~mm}^{2}$; and theoretical pixel size: $0.18 \times 0.18 \mu \mathrm{m}^{2}$. (f) Magnification: $100 \times 100$; FOV: $0.28 \times 0.28 \mathrm{~mm}^{2}$; and theoretical pixel size: $0.14 \times 0.14 \mu \mathrm{m}^{2}$.

sample and the scintillator has to be $300 \mathrm{~mm}$ at least, hence, Fresnel diffraction is to be expected. Such a phenomenon can be observed in Fig. 9(d) at the upper part of the trabecula border, where at least one interference fringe can be detected with the bare eye. As described in Ref. 9, the microscope produces in-line holographic images: the edge-enhancing imaging is, therefore, easily accessible and, if numerical phase retrieval is provided, ${ }^{20}$ quantitative holotomography can also be performed. Exposure time for the images depicted in Fig. 9 ranges from $1 \mathrm{~s}$ for the $20 \times 20$ magnification to $30 \mathrm{~s}$ for $100 \times 100$. It has, however, to be mentioned that those measurements had been made with a preliminary setting of the 
beamline. In the near future, the ring current will be doubled and the wiggler gap closed to $7.5 \mathrm{~mm}$, resulting in a fivefold reduction of the exposure times. It has been shown that the Bragg magnifier can produce hard $\mathrm{x}$-ray $2 \mathrm{D}$ radiographic images with magnification factors of $20 \times 20$ to $100 \times 100$ and exposure times of few seconds. Therefore, its integration in synchrotron-based tomographic investigations represents the immediate development.

\section{ACKNOWLEDGMENTS}

The authors thank B. Patterson, D. Vermeulen, and M. Lange for their generous help in setting up the beamline. The authors appreciated the efficient collaboration with B. Lux and Dr. V. Alex from the Institut für Kristallzüchtung, Berlin. The authors thank Professor H. Grimmer and Dr. D. Clemens of PSI for their help with the preliminary characterization of the crystals. This work has been supported by the ETH Board.

${ }^{1}$ U. Bonse and F. Busch, Prog. Biophys. Mol. Biol. 65, 133 (1996).

${ }^{2}$ H. R. Lee, B. Lai, W. Yun, D. C. Mancini, and Z. Cai, Proc. SPIE 3149, 257 (1997).

${ }^{3}$ B. Dowd, G.H. Campbell, R.B. Marr, V.V. Nagarkar, S.V. Tipnis, L. Axe, and D.P. Siddons, Proc. SPIE 3772, 224 (1999).
${ }^{4}$ T. Weitkamp, C. Raven, and A.A. Snigirev, Proc. SPIE 3772, 311 (1999).

${ }^{5}$ A. Koch, C. Raven, P. Spanne, and A. Snigirev, J. Opt. Soc. Am. A 15, 1940 (1998).

${ }^{6}$ B. Lai et al., Rev. Sci. Instrum. 66, 2287 (1995).

${ }^{7}$ C. Schroer et al., Proc. SPIE 4503, 23 (2002).

${ }^{8}$ M. Kuriyama, R.C. Dobbyn, R. Spal, H.E. Burdette, and D.R. Black, J. Res. Natl. Inst. Stand. Technol. 95, 559 (1990).

${ }^{9}$ R. Spal, Phys. Rev. Lett. 86, 3044 (2002).

${ }^{10}$ A. Authier, Dynamical Theory of X-ray Diffraction (Oxford University Press, New York, 2001).

${ }^{11}$ S. Brennan and P. Cowan, Rev. Sci. Instrum. 63, 850 (1992).

${ }^{12}$ Computation performed with the in-house developed software XDF (to be published).

${ }^{13}$ M. Sanchez del Rio and R. Dejus, Proc. SPIE 3448, 230 (1998).

${ }^{14}$ R. Caciuffo, S. Melone, F. Rustichelli, and A. Boeuf, Phys. Rep. 152, 1 (1997)

${ }^{15}$ W. H. Zachariasen, Theory of X-Ray Diffraction in Crystals (Wiley, New York, 1945)

${ }^{16}$ B. D. Patterson, R. Abela, and J.F. van der Veen, Chimia 55, 534 (2001).

${ }^{17}$ M. Stampanoni, G.L. Borchert, R. Abela, B. Patterson, D. Vermeulen, P. Rüegsegger, and P. Wyss, Acta Phys. Pol. B 33, 463 (2002).

${ }^{18}$ M. Stampanoni, G.L. Borchert, P. Wyss, R. Abela, B. Patterson, S. Hunt, D. Vermeulen, and P. Rüegsegger, Nucl. Instrum. Methods Phys. Res. A 491, 291 (2002)

${ }^{19}$ V. V. Nagarkar, T.K. Gupta, S.R. Miller, Y. Klugerman, M.R. Squillante, and G. Entine, IEEE Trans. Nucl. Sci. 45, 492 (1998).

${ }^{20}$ P. Cloetens, Appl. Phys. Lett. 75, 2912 (1999). 